\title{
SINGLE-PLLSE INTENSITY FLUCTUATIONS BY CROSS-CORRELATION WITH LONGITUDE OF THE AVERAGE PROFILE
}

\author{
M .V. Popov \\ P. N. Lebedev Physical Institute, Academy of Sciences \\ W. SIEBER \\ Fachhochschule Niederrhein Fachbereich Electrotechnik
}

\section{Introduction}

Single-pulse radio emission from pulsars has been observed to vary both in intensity and position inside the pulse window. In some cases the variations show a rather regular structure (drifting subpulses). Such a regular structure can clearly be studied by fluctuation spectral analysis. The analysis has identified some particular peculiarities in the fluctuation spectra at different longitudes of the pulse window in many pulsars (Backer 1973, Page 1973, Popov and Smirnova 1982). In pulsars with complex integrated profiles (PSR 1919+21, PSR 1237+25) narrow features in the fluctuation spectra vary strongly with longitude, at some longitudes being quite undistinguishable. The fluctuation spectra corresponding to the autocorrelation functions of intensity fluctuations do not provide information on the mutual dependence of these fluctuations at different longitudes. To investigate such a dependence we propose a cross-correlation analysis of the intensity fluctuations at different longitudes of the pulse window. In this paper we present the results of the cross-correlation analysis for 12 pulsars. Single-pulse observations were conducted at $1700 \mathrm{MHz}$ with the Effelsberg $100-\mathrm{m}$ radio telescope during the years 1978-1982.

\section{Data processing}

The observed intensity of a pulsar's radio emission varies with longitude (phase). within the period and pulse number. The raw observations were, therefore, preprocessed to form a two-dimension array $I_{i}(k)$, where $k$ enumerates the pulse number $(1 \leq k \leq N)$, and $i$ identifies the longitude bin $(1 \leq i \leq 32)$ within a given period. $I_{i}(k)$ represents the intensity integrated over a range of longitude after subtraction of the baseline (off-pulse intensity). We then computed the cross-correlation coefficients between intensities at different longitudes and pulses according to

$$
C_{i, j}(\mu)=\frac{1}{\sigma_{i} \sigma_{j}(N-\mu)} \sum_{m=1}^{N-\mu} I_{i}(m) I_{j}(m+\mu)-\bar{I}_{i} \bar{I}_{j}
$$

where $\sigma_{i}$ designates the square root of the variance of $I_{i}(m)$ taken at the longitude bin $i$ and computed over $N$ successive pulses. $\bar{I}_{i}$ denotes the mean over $N$ pulses, and $\mu$ represents the lag in pulse number.

Table 1 summarizes the instrumental parameters of the observations analyzed - that is, the total number of pulses used in the analysis for each pulsar and the resolution in longitude.

Table 1 Number of pulses and longitude resolution

\begin{tabular}{|ccl|}
\hline PSR & $\mathrm{N}$ & $\Delta \phi^{\circ}$ \\
\hline $0031-07$ & 7000 & 1.5 \\
$0329+54$ & 2000 & 0.8 \\
$0809+74$ & 8000 & 1.1 \\
$0823+26$ & 4000 & 0.33 \\
$0950+08$ & 1000 & 6.3 \\
$1133+16$ & 2000 & 0.39 \\
$1237+25$ & 1000 & 0.46 \\
$1508+55$ & 1536 & 0.45 \\
$1604-00$ & 4000 & 0.6 \\
$1642-03$ & 2000 & 0.65 \\
$1919+21$ & 8000 & 0.52 \\
$2020+28$ & 7000 & 0.62 \\
\hline
\end{tabular}

\section{Results}

We present the results of our analysis for five selected pulsars in figures which each have five subpanels. The first three panels of each figure show the cross-correlation coefficients $C_{i j}(\mu)$ in contour diagram maps with $\mu=0$ at the top (a), $\mu=1$ in the middle (b), and $\mu=2$ at the bottom (c). Lines of constant correlation are drawn from a level of 0.05 to 0.7 in steps of 0.05 , negative correlations are indicated by dashed lines. The $X$ and $Y$-axes are scaled in degrees of longitude (same scale for both axes); we have also drawn the integrated profiles on both axes for easy comparison. The second and third panels of every figure show the crosscorrelations between successive pulses $(\mu=1)$ and pulses which are separated by two periods $(\mu=2)$. For these panels the $X$-axis always designates the longitude of the earlier pulse and the $Y$-axis the longitude of the later, following pulse. 


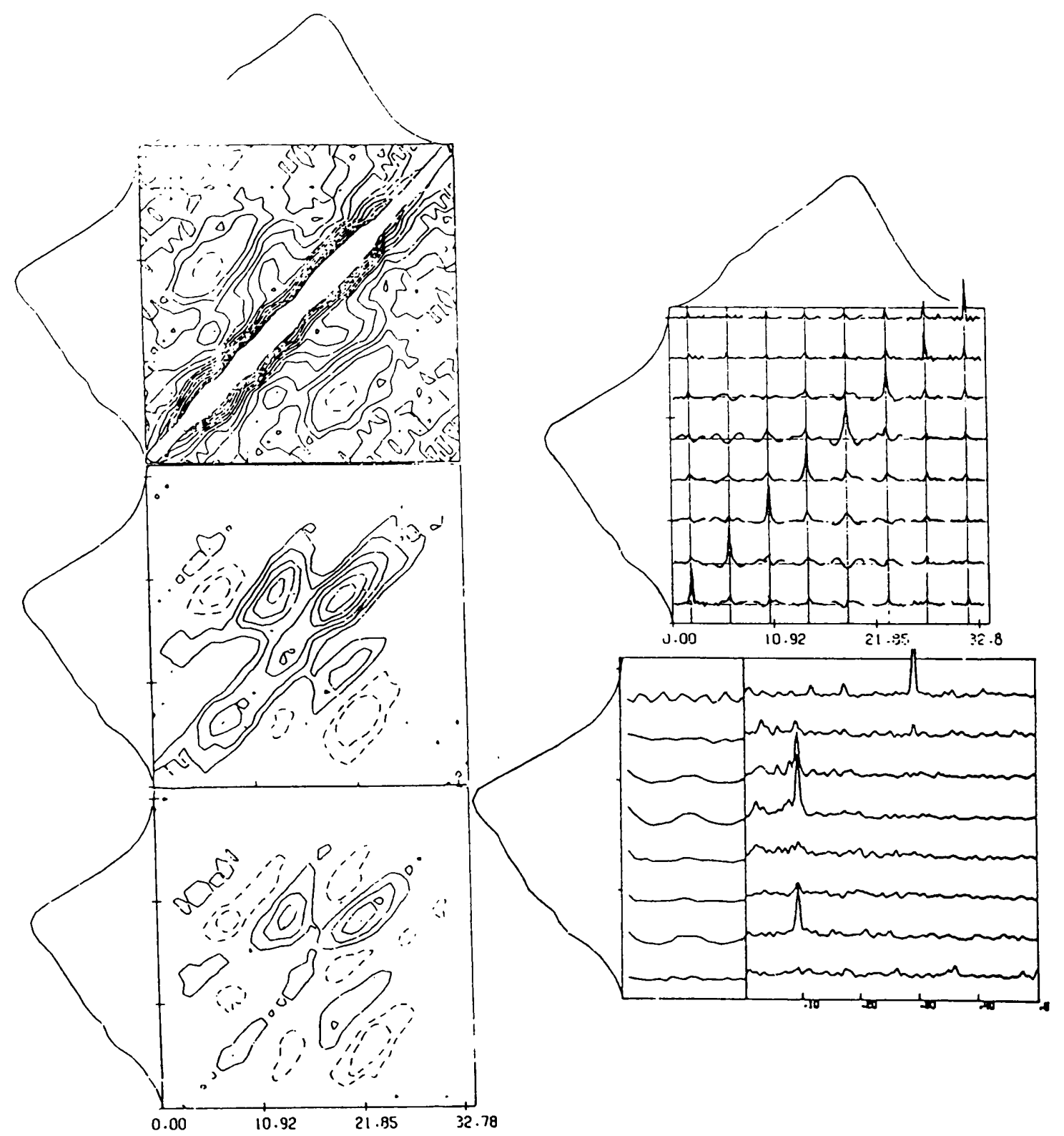

Figure 1 PSR $0809+74$. The integrated pulse of this pulsar can be classified as a conal profile, and the angle $\beta$ is thought to be greater than $5^{\circ}$. PSR $0809+74$ is well known for its regular drift of subpulses from the trailing to the leading edge of its integrated pulse profile with $P_{3}=11 P_{1}$ and $P_{2}=25 \mathrm{~ms}$ (Bartel et al. 1981) at $1700 \mathrm{MHz}$. The pattern shown in the figure reflects this behavior quite nicely. It demonstrates that the subpulse drifting is more developed near the edges but not at the extreme boundary of the pulse profile. (The narrow feature at a frequency of $0.31 / P_{1}$ in the fluctuation spectrum [panel e] at the trailing edge of the profile is an alias of a $50-\mathrm{Hz}$ harmonic.)

It is interesting to note that the correlation does not usually vanish for pulses which are two periods apart $(\mu=2)$. Therefore, at the top right of each figure (panel d) we show the cross-correlation functions for a limited range of periods $(-8 \leq \mu \leq+8)$. The cross-correlation functions are shown there at selected longitudes only ( 8 for each pulse) as a function of $\mu$, the lag in periods. The bottom right of each figure (panel e) shows the first few lags (20) of the autocorrelation function along the corresponding longitude interval, and on the right one may find the fluctuation spectra of the intensity variations.

Overall, the figures reveal a great variety of behavior. It therefore seems appropriate to give a short. descrintion of the nesuliarities of the individ-

ual pulsars and then to try to obtain some general conclusions. The pulsars have been arranged in order of decreasing $\beta$, the angle between the line of sight and the magnetic axis of the pulsar. The values of $\beta$ were taken from Lyne and Manchester (1988). In this order we might expect-according to the classification scheme of Rankin (1986)-first to find pulsars which have only conal emission and then those with both core and conal emission.

\section{Conclusions}

From the material presented above we may draw the following general conclusions: 


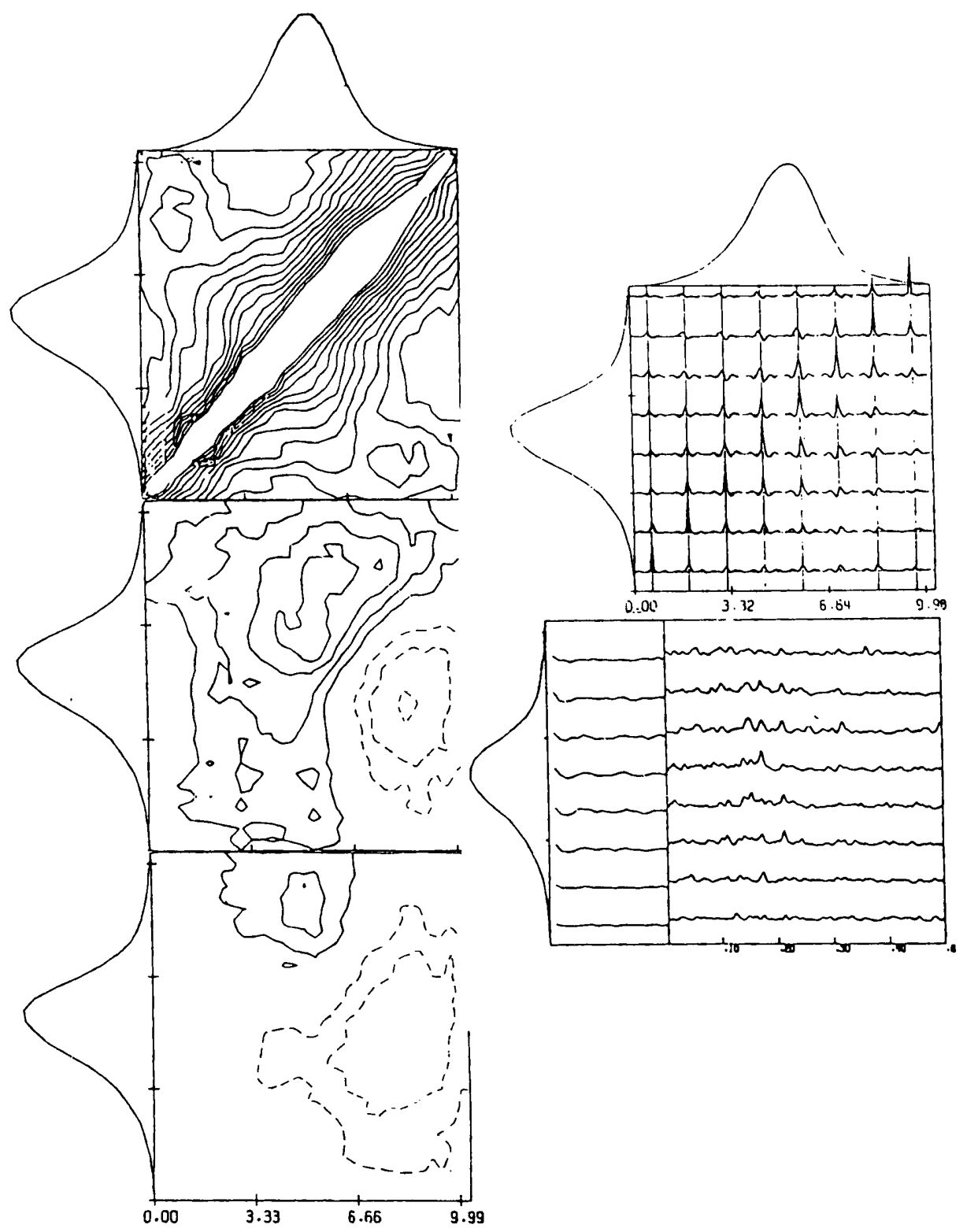

Figure 2 PSR 0823+26. Lyne and Manchester (1988) estimate $\beta$ to be about $5^{\circ}$. The fluctuation spectra for this pulsar (panel e) does not display narrow line features. One can, however, clearly identify a systematic intensity modulation with $P_{3}=6 P_{1}$ from panel (d). The modulation seems, however, to die away in a time of the order of the $P_{3}$ itself. The regions of correlation are broad, covering nearly the whole longitude range of the main pulse. We think that PSR $0823+26$ may be considered as a transition from pulsars with cone-dominated emission to those with core emission.

1. Each pulsar has its own characteristic crosscorrelation map which describes the mutual dependence of intensity fluctuations at different longitudes of pulse profile. The correlation map seems to be stationary when more than about 1000 periods are analyzed.

2. For pulsars with single-cone-type profiles, we usually find very similar cross-correlation patterns reflecting the drifting subpulse phenomenon (PSR 0809+74, PSR 0031-07, PSR 0823+26).

3. Pulsars with double-cone-type profiles show distinct longitude ranges of increased cross-correiation. The cross-correlation times are determined by a periodic modulation process which may produce different phase shifts at different longitudes (PSR 1133+16, PSR 1919+21).
4. The cross-correlation maps are usually separated into two main regions for pulsars with a core component in the profile. The intensity fluctuations at the leading part of profile show no correlation (or even some anticorrelation) with the intensity fluctuations at the trailing part of profile (PSR 0329+54, PSR 1604-00, PSR 1237+25).

5. The cross-correlation functions have time scales of about $20 P_{1}$ (slow variation) in the saddle region of pulsars with double profiles and in the neighborhood of core components. The regions with this type of pattern often appear to be shifted from the center to the leading part of the profile (PSR 1133+16, PSR 2020+28, PSR 1508+55, and PSR 1237+25).

6. Pulsars with complex integrated pulse pro- 

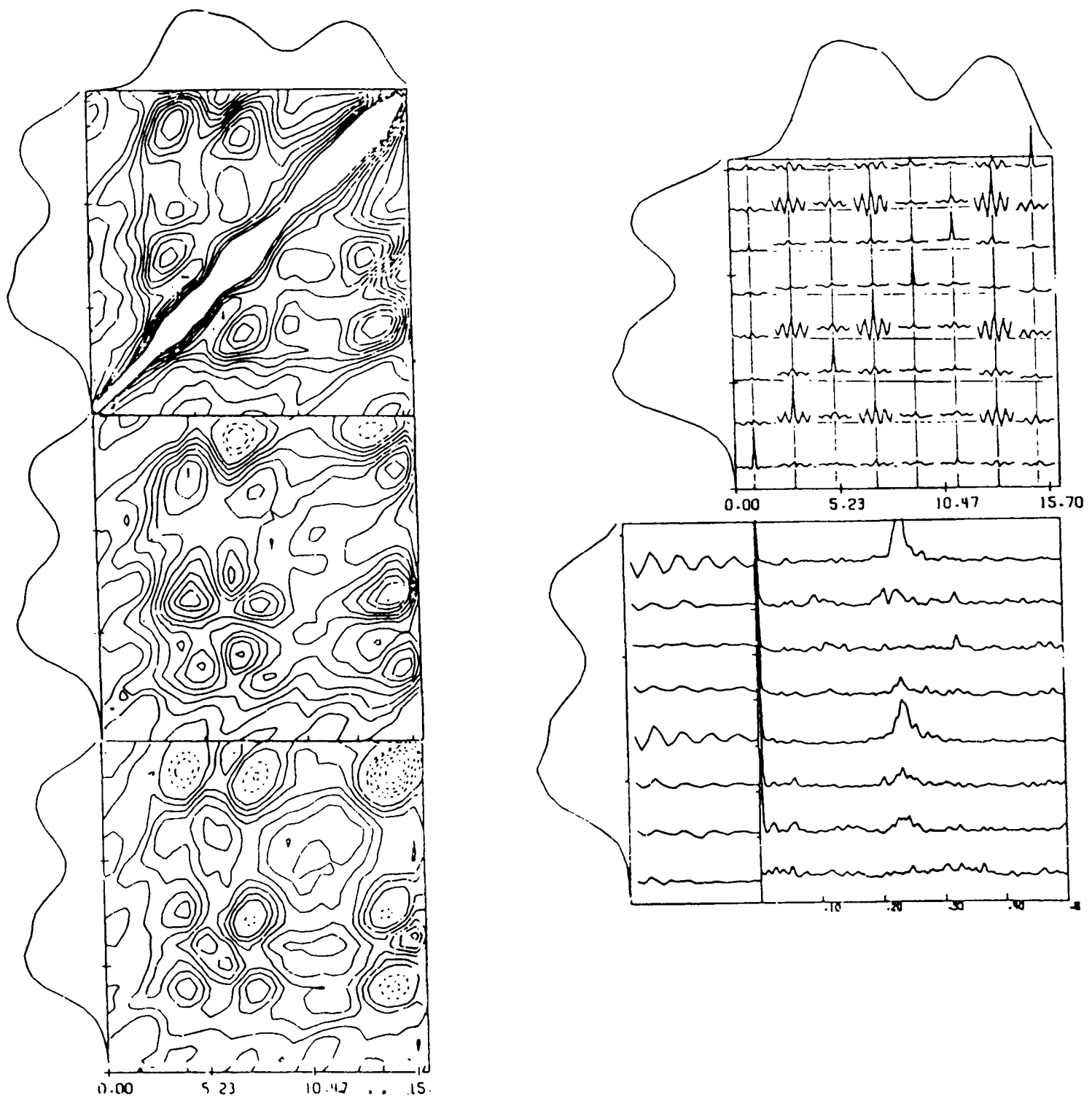

Figure 3 PSR 1919+21. $\left(\beta=4.1^{\circ}\right)$. The cross-correlation maps show a somewhat patchy appearance, one may however clearly distinguish three longitude ranges which show strong mutual correlation. Panel (d) demonstrates that this correlation is caused by an intensity modulation at just those longitude intervals with $P_{3}=4 P_{1}$. The modulation is in-phase at the edges of the pulse window, but there is a phase shift of one period between the outer and central longitude intervals. The entire pattern is produced by a system of drifting subpulses which was also carefully studied by Backer (1973), Cordes (1975), and Wolszczan and Proszynski (1986). It is interesting to note that in the longitude range near the saddle of the mean-pulse profile, no intensity correlation if found with other longitude intervals of the pulse window. The enhanced correlation extending over many periods which can be seen over the greater part of the pulse window may well be caused by scintillations.

files show regions of correlation with time scales less than one period (fast variations). The radio emission at these longitudes does not correlate with the emission at any other longitude of the pulse window (PSR 1919+21, PSR 1604-00).

7. Many pulsars show enhanced correlation between the extreme edges of the pulse window. The outstanding example of this phenomenon is PSR 0950+08, where the correlation is about $50 \%$ between the edges of the main pulse and the interpulse (which are thought to originate from the same magnetic pole). Other examples are PSR 0809+74, PSR 0031-07, PSR 2020+28, and PSR 1604-00.

\section{Interpretation}

The general outline of the polar-cap model may be summarized in the following few sentences: In the polar region of open magnetic field lines (MFL) of a rotating neutron star, a huge potential drop can be developed by the rotation-induced electric field. It appears that this potential drop is sufficiently large to produce, through pair creation, a dense outflowing stream of relativistic plasma which (being unstable by some plasma instability) can bunch appropriately to give coherent microwave radiation at some height above the neutron-star surface. 

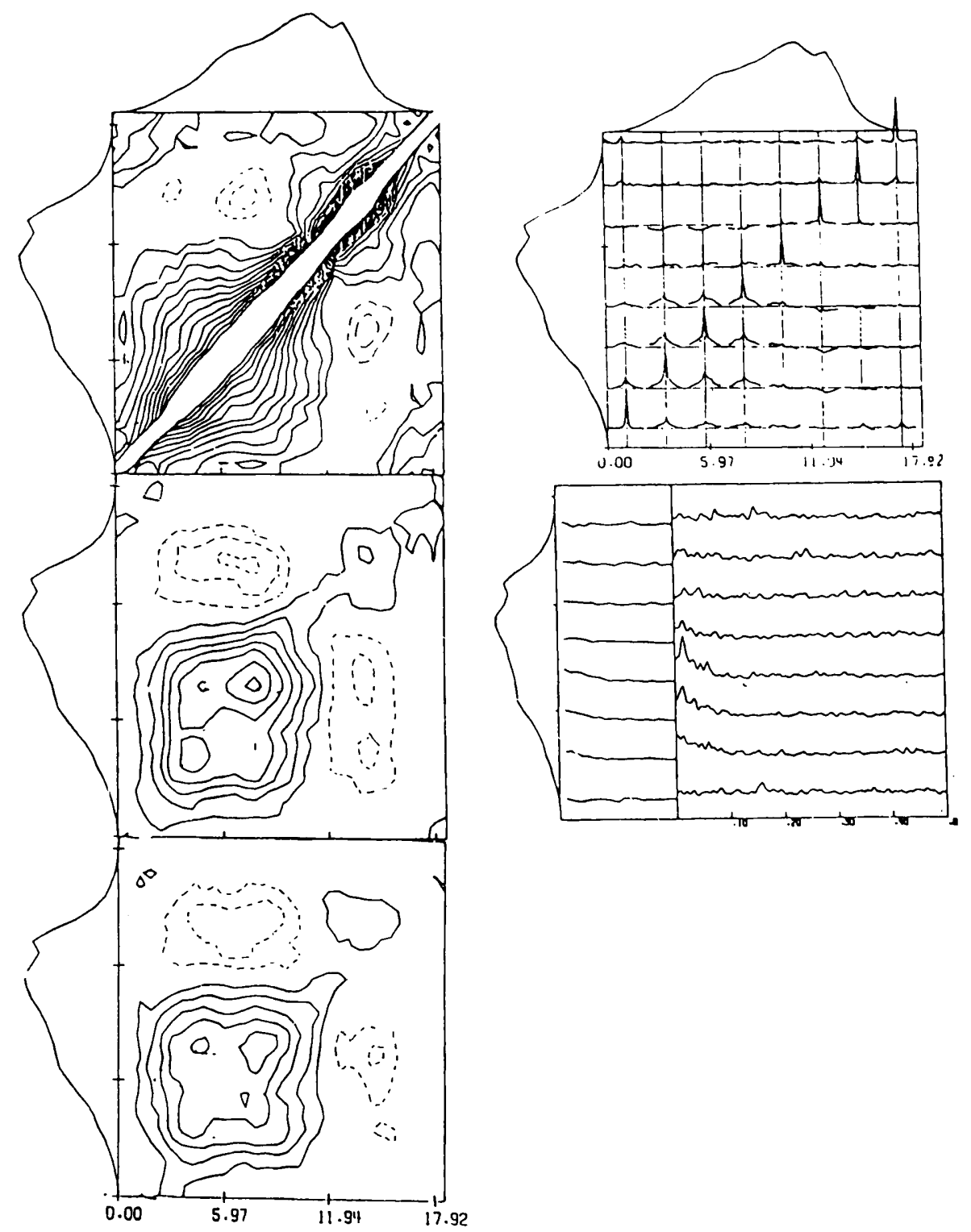

Figure 4 PSR 1604-00. The integrated profile seems to consist of three components. In the observations of Morris et al. (1981), the change of sense of circular polarization occurs near the longitude corresponding to the maximum intensity of the average profile, as is typical of the core type of emission (Rankin 1986). The angle $\beta$ then appears to be rather small, and we identify this object as a core-dominated pulsar. The cross-correlation map is separated into two regions: the extensive region covering the first two-thirds of the integrated profile from its beginning and a second narrow region corresponding to the last component of the profile. The first region has a long time correlation (time scale is greater than $20 P_{1}$ ), and the second one shows a shorter time scale of about $2 P_{1}$. Probably the first component is a core component, and the second is a conal one. There is also some extra correlation between the edges of pulse window.

In the polar-cap models the so called "spark" discharge providing the avalanche production of electron-positron pairs can be developed in at least two ways: (1) electron-positron creation from the curvature emitted $\gamma$-photons of electrons and positrons accelerated in the polar-cap potential drop (Sturrock 1971); and (2) pair creation by the thermal X-rays emitted from "hot spots" in the polar cap in the Coulomb field of relativistic ions accelerated in the gap.

The Sturrock mechanism of pair production has a quite particular geometric structure (Cheng and Ruderman 1977b). In a dipole magnetic field the discharge spark moves progressively to the magnetic axis and vanishes there. The spatial structure of the resulting electron-positron stream will be a narrow vertical sheet located in the meridional plane of the magnetic field. The time necessary for the spark to cross the polar cap is about $100 \mu s$ (Cheng and Ruderman 1977b). Thus, pure Sturrock discharge rapidly dies away and must be continually restarted. The discharge re-ignition may be provided by pair creation from thermal X-rays, emitted from locally heated patches on the stellar surface. The subsequent pair creation occurs in the Coulomb fields of relativistic ions. The strong local heating is sup- 

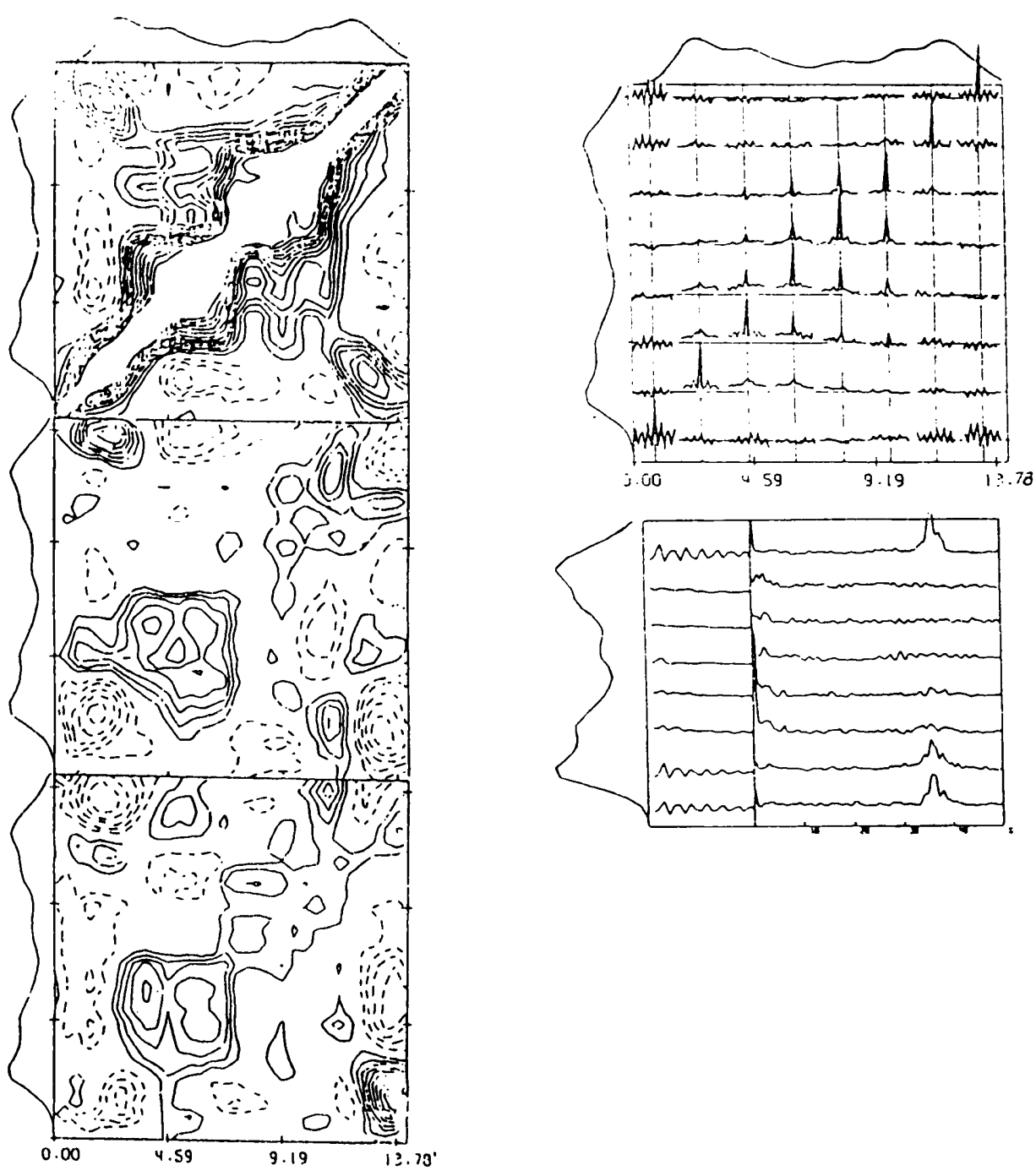

Figure 5 PSR 1237+25. In our sample this pulsar represents the closest traverse of the sight line relative to the neutronstar magnetic axis. The value of $\beta$ is $0.9^{\circ}$. The integrated profile consists of five components and has a symmetrical appearance. The cross-correlation map for zero lag $(\mu=0)$ is also quite symmetrical. It is separated into four regions: two narrow regions at the edges and two broader regions in the central longitudes of profile. There is also significant correlation between the edges of pulse window. One can see in figure $5(\mathrm{e})$ that a considerable range of longitude is intensity modulated with a period of $P_{3}=? .8 P_{1}$ and that the modulation decreases strongly in the middle of pulse window. The modulation completely vanishes in the corresponding fluctuation spectra, but the cross-correlation functions still detect this modulation at all longitudes. At central longitudes, especially to the right of the middle, the cross-correlation functions show rapid intensity fluctuations with time scales of less than one period. To the left of this point slow variations of intensity are observed with time scales of about $20 P_{1}$.

plied by an intense flux of $10^{12} \mathrm{eV}$ electrons produced in the discharge and directed into the surface. Another possibility to sustain the discharge is the shower produced by the impact of high energy electrons on the stellar surface. The shower contains a few photons with energies above $1 \mathrm{MeV}$ which, being emitted from the surface, can initiate pair production in the region of the discharge (Jones 1979). Since in both cases the direction of the emitted photons is not connected with the magnetic field geometry, the discharge can be restarted anywhere, including the original field line. Now the spatial structure of the discharge will not represent a thin sheet, but the discharge will occupy some limited tube of field lines extending from the point of ignition to the magnetic axis.

Now we shall attempt to compare the results of our cross-correlation analysis with a theoretical understanding of the geometry and dynamics of pairproduction discharges in polar-cap models of pulsar radio emission.

The circles in figure 6 outline the polar-cap region at the stellar surface, the center being the magnetic pole. Dashed elongated regions $a, b, c, d$, and 

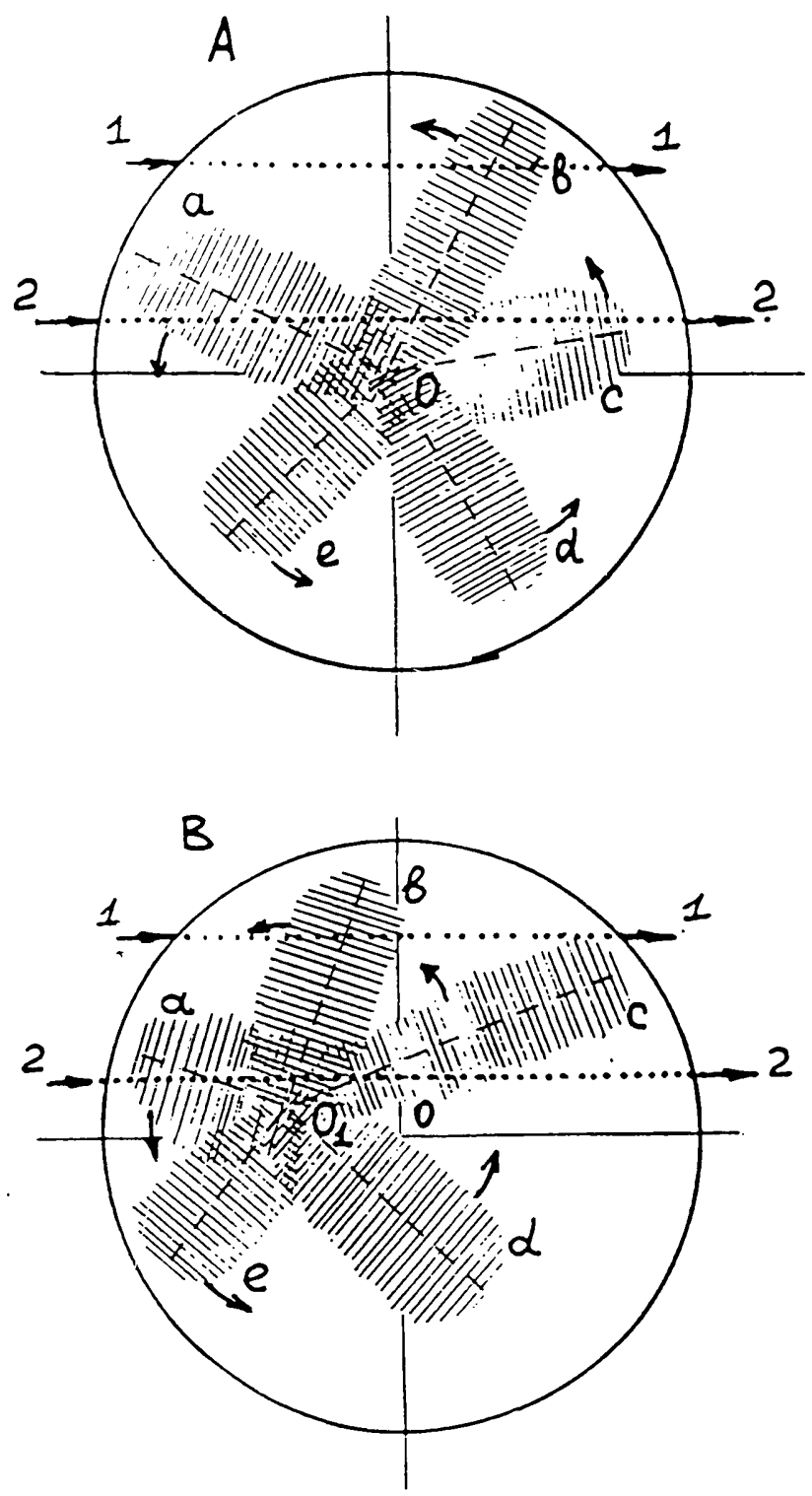

Figure 6 Diagram of the polar-cap region at the stellar surface, the center being the magnetic pole. Dashed elongated regions $a, b, c, d$, and $e$ represent the discharge regions. The arrows near the dashed regions show the direction of the drift. Dotted lines 1-1 and 2-2 show the projections of the line of sight. In the lower diagram the magnetic pole is displaced from the center of the polar cap.

$e$ represent the discharge regions where Sturrock's mechanism of pair creation plays a considerable role. So, all the regions are located along the magnetic meridian. They start at some magnetic latitude and always finish at the center. Dashed lines show two versions of the observer's sight-line trajectory, projected onto the polar-cap region along the dipole magnetic field lines from the region of radio emission. The arrows near the dashed regions show the direction of the drift around the magnetic pole. It is suggested that when the sight line intersects the discharge region the observer will see a subpulse of radio emission.
This simplified diagram already provides an explanation of some of the results obtained. In fact, for pulsars with a conal profile (sight-line trajectory 1) one or two subpulses can be observed; they are located at a definite distance from each other and occupy a limited range of longitude. The subpulses will be drifting in the proper direction if the discharge regions exist for a sufficient time interval (PSR 0809+74, PSR 0031-07).

A more complex pattern will be observed for pulsars with core components in the average profile (sight-line trajectory 2). The line-of-sight trajectory for such cases passes through several discharge regions converging to the magnetic pole. The discharge regions, located at opposite parts of the polar cap relative to the magnetic pole, may be activated independently, and the mutual correlation at the respective longitudes can be very small. At the longitudes of conal components the observer will see a periodic modulation of radio emission which is approximately in phase over a considerable range of longitude for each component instead of the regular drift observed in the case of trajectory 1 . Because of the convergence of all the discharge regions to the magnetic pole, the periodic modulation at the central longitudes will be strongly smoothed, and the time scale of the intensity fluctuations at those longitudes will increase due to the continuous contribution from any of the existing discharge regions to the subpulse intensity (slow variations). Such characteristics have been obtained in our analysis for pulsars with core components in the integrated profile (PSR 0329+54, PSR 1604-00, PSR 1237+25). However, both the boundary of mutual correlation and the longitudes of slow variations often appear to be shifted from the middle of the integrated profile, usually in the leading direction. This fact may be explained by assuming that the geometry of the magnetic field in the region of plasma formation is different from a pure dipole. The possibility is illustrated in figure 6(b), where the position of the local magnetic axis is shifted from the center of the polar cap due to the contribution of a quadrupole component of the field. Because of the almost tangential emission of the $\gamma$-ray curvature radiation which produce the electron-positron pairs by the Sturrock mechanism, the discharge always moves outward on the convex side of the field lines. So the position of a new local magnetic axis should be the center of our correlation map. The existence of a single local magnetic pole in a region of copious pair production leads to the conclusion that the magnetic field in this region has a rather simple geometry; numerous multipoles do not seem to be present in such a region. 